\title{
Instrumenting Hand-Held Surgical Drills with a Pneumatic Sensing Cover for Haptic Feedback
}

\author{
Chiara Gaudeni ${ }^{1(\otimes)}\left(\mathbb{D}\right.$, Tommaso Lisini Baldi ${ }^{1}$ (D), Gabriele M. Achilli ${ }^{2}$, \\ Marco Mandalà ${ }^{3}(\mathbb{D})$, and Domenico Prattichizzo ${ }^{1,4}$ (D) \\ 1 Department of Information Engineering and Mathematics, University of Siena, \\ Siena, Italy \\ \{gaudeni,lisini,prattichizzo\}@diism.unisi.it \\ 2 Department of Engineering, University of Perugia, Perugia, Italy \\ 3 Department of Medicine, Surgery and Neuroscience, University of Siena, \\ Siena, Italy \\ 4 Department of Advanced Robotics, Istituto Italiano di Tecnologia, Genoa, Italy
}

\begin{abstract}
Despite the recent achievements in the development of open surgery tools, preserving the haptic capabilities during drilling tasks is still an open issue. In this paper, we propose a novel tool for handheld drills composed of a cover for force sensing and a haptic display for force feedback. A pneumatic device has been developed to estimate the contact force occurring during the interaction between drill bit and bones. A performance comparison with a precise commercial force sensor proved the reliability of the measurements. A haptic ring is in charge of providing cutaneous sensations helping the surgeon in performing the task. The effectiveness of our method has been confirmed by experimental results and supported by statistical analysis.
\end{abstract}

\section{Introduction}

Technological advancements in surgical tools have expanded the field of robotassisted surgery to newer specialties. Even if the achievements in the last years have been impressive, current robotic surgical systems are still limited by the lack of haptic feedback. It has been proved that restoring the haptic capability in robotic surgery contributes to improve accuracy and safety in performing complex and delicate surgical tasks [9]. On the other hand, also open surgery may suffer from a reduction of tactile perception. In fact, even if in these procedures surgeons directly interact with the patient's body, some surgical tools, e.g. drills, may limit the haptic perception. As a matter of fact, a common issue in surgical drilling is that vibrations generated by the tool affect the perception of the surgeon, reducing, for instance, the capability in discerning different tissues and detecting the break-through force [5].

In this paper, we focus on otologic procedures, where a precise control of the surgical drill is required because the critical anatomy within the middle ear, 


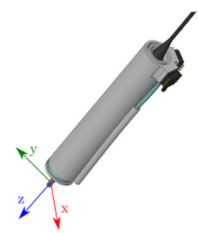

(a)

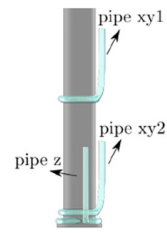

(b)

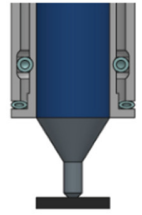

(c)

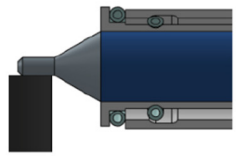

(d)

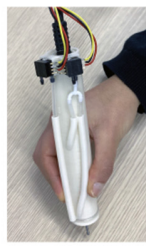

(e)

Fig. 1. The developed sensing system: (a) CAD model; (b) attachment of pipes to the inner shell; (c)(d) details of the sensing mechanism measuring perpendicular and tangential forces, respectively; (e) a user holding a surgical drill enriched with the sensing cover. Outer soft silicone pipes are covered by rigid housings to prevent the surgeon from touching them and affecting the measurements.

inner ear, and skull base can be accessed by drilling within the temporal bone for operations which demand high precision and accuracy [8]. Several researchers pointed out benefits of restoring haptic feedback in hand-held drilling procedures. In [1], force sensing has been elected as the appropriate way to obtain controlled penetration in the patient's body and automatic discrimination among layers of different tissues. Hessinger et al. integrated a thrust force sensor into the drill to enable high accuracy during pedicle screw positioning [3]. In the aforementioned works, the force measure is obtained integrating sensors into the tool mechanism. In this paper, we propose a pneumatic method to measure the contact force between the drill bit and the bone without modifying the internal structure of the tool. The aim is to create an instrumented cover that can be easily customized and adapted to the off-the-shelf hand-held drills. Moreover, the proposed system is capable of rendering the force feedback to the user by means of a haptic ring. To the best of our knowledge, this represents the first attempt to assist a surgeon with haptic feedback in open surgery without modifying the existing equipment.

\section{Design of the Pneumatic Force Sensor}

\section{Working Principle}

Measuring contact forces between the drill bit and a surface without modifying existing tools encounters several non-trivial challenges. Because of the drilling task, the sensing system has to be placed far from the contact point. Common precise and accurate 3 -axis force sensors are bulky and not suitable for small devices. Thus, we developed a pneumatic system capable of estimating forces using pipes and air pressure sensors. The great advantage of using a pneumatic system is that it is lightweight, tiny, and measurement information is transferred by means of a gas to the sensors, which can be located out of the operational workspace. We exploit a sensing structure consisting of two concentric cylindrical shells separated by a gap, as shown in Fig. 1a. The inner shell (Fig. 1b) is rigidly attached to the body of the drill, while the outer shell is the one held by the surgeon. Soft silicone pipes are placed between the two shells to fill the gap, 
preventing any relative movements when no forces are applied. They represent also the sensing element of the device, as explained below.

The working principle of the developed sensing system relies on the assumption that the drill-hand system is under mechanical equilibrium conditions until an external force is applied to the drill bit. When the drill bit comes into contact with the bone, the inner shell moves towards the outer shell along the direction of the contact (see Figs. 1c and 1d). This displacement generates a compression of soft silicone pipes depending on the external force. In the considered surgical scenarios, torque components are treated as negligible, because the drill bit does not enter deeply into the bone generating significant values of torque. It is worth noting that the grasp squeezing forces applied by the surgeon do not cause any structural deformation of the pipes thanks to the high stiffness of the external shell. Then, the only deformations are due to the forces applied to the drill bit. To estimate the entity of the compression, the increase of pipes internal pressure is measured by means of air pressure sensors placed outside the outer shell. Pipes are not additionally inflated: at the steady state they are at the equilibrium with the external air pressure.

\section{Hardware Implementation}

As depicted in Fig. 1e, we present a proof of concept in which the pneumatic force sensor is composed of two 3D-printed parts made of ABSPlus (Stratasys Inc., USA), soft silicone pipes (ID $2.5 \mathrm{~mm}$, OD $3.5 \mathrm{~mm}$ ), and two $2 \mathrm{kPa}$ differential pressure sensors (MPXV7002DP, NXP Semiconductors, NL) measuring forces along the $\mathrm{z}$-axis and on the xy-plane, respectively (as in the reference system of Fig. 1a). The total weight of the cover is $51 \mathrm{~g}$. Each pipe is ring-shaped and firmly attached to the inner shell as shown in Fig. 1b, with one end leak-proof sealed. The opposite side of the pipe conveys the pneumatic information to the pressure sensors. Forces on the xy-plane are measured by a sensor connected to pipes $x y$. Two pipes spaced along the length of the drill are required to prevent possible relative movements between the two shells when no forces are applied. It is important to notice that each pipe goes out of the outer shell as soon as the loop close, through a small hole. In this way, the measurement resolution is the same in all the directions of the xy-plane. Both the shells contain grooves for enclosing the pipes so as to reduce the width of the device. In this way, the gap between the shells corresponds to the internal diameter of the pipes. In the outer shell, the cavity is vertically extended so as not to detect pressure variations when the only force involved is along the z-axis. Taking advantage of the flange on the lower part of the shell, it is possible to measure with a second pressure sensor the forces exerted on the z-axis by means of pipe $z$ (see Fig. 1b). The effect of gravitational force has been considered negligible with respect to the forces at work, due to the lightweight of the drill. Moreover, we supposed that in a such accurate task the surgeon compensates the weight by his hands and the contact force is equivalent to the force exerted by the surgeon. Vibrations generated by the drill are filtered by means of a hardware $\mathrm{R}-\mathrm{C}$ filter with an experimentally selected cut-off frequency of $144.68 \mathrm{~Hz}$. To further isolate sensors from vibrations, two tiny sponge layers are placed under the sensor housings. 


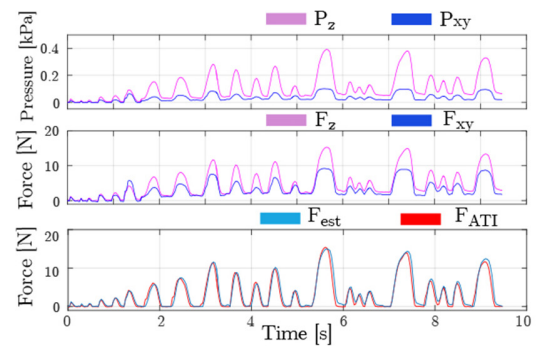

(a)

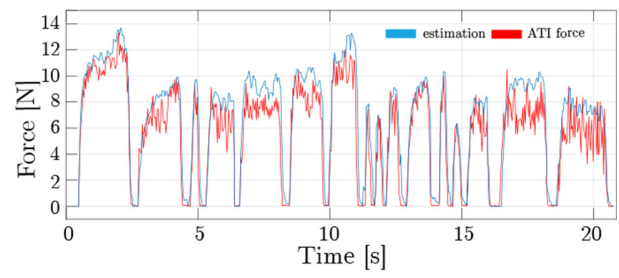

(b)

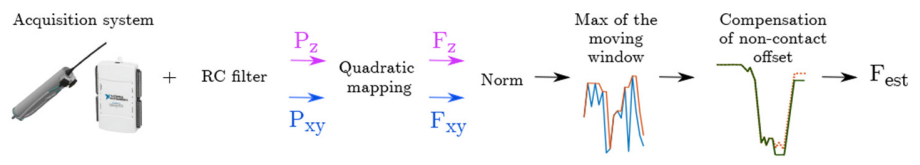

(c)

Fig. 2. Force estimation. In (a) raw pressure values, the correspondent forces, and the comparison between the final force estimation and the ground-truth value are reported. Values obtained in a representative trial, affected by vibrational noise, are in (b). In (c), the steps of the force estimation are detailed.

Analog data from the sensors are acquired using a NI USB-6218 DAQ. Finally, a software algorithm, described below, processes the signal.

\section{Software Implementation}

A calibration procedure is required to correctly transform data from pressure sensors into forces. The initial calibration and the subsequent validation phase are performed with the drill switched off. A high precision ATI Gamma F/T sensor (ATI Industrial Automation, USA) is used to identify the force-pressure relation. A separate procedure is required for calibrating the two sensors. For what concerns the z-axis calibration, the drill was vertically pushed toward the ATI sensor for a total of 50 contact actions, so that the generated force deforms only pipe $z$, as depicted in Fig. 1c. In accordance with [2], data gathered from the pressure sensor and the ATI were quadratically interpolated using a Matlab ${ }^{\circledR}$ algorithm. The same procedure was repeated for the xy-plane, keeping the drill horizontally (Fig. 1d). For the tool exploited in this work, the two found relations are: $F_{z}=5034 \cdot P_{z}-2280 \cdot P_{z}^{2}$ and $F_{x y}=9542 \cdot P_{x y}-1017 \cdot P_{x y}^{2}$, being $P_{x y}$ and $P_{z}$ the pressure values. As a final step, the norm of $F_{z}$ and $F_{x y}$ is computed. Indeed, from the user point of view, there is no need to distinguish the three components of the force: the surgeon just needs to have a feedback on the total force exerted, which corresponds to the norm of $\mathrm{F}_{z}$ and $\mathrm{F}_{x y}$. As noticeable in Fig. 2a, this value is affected by the relatively slow dynamic of the pneumatic system. After breaking contact, the pressure of the pipes does not immediately reach the initial zero-value. A rapid decrease of the pressure is followed by a slow downwelling. Thus, we introduced a compensation algorithm which brings to zero the "non-contact offset", identified as a flat trend after a significant negative slope. 


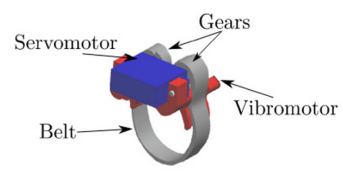

(a)

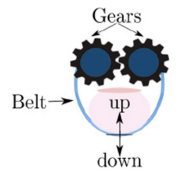

(b)

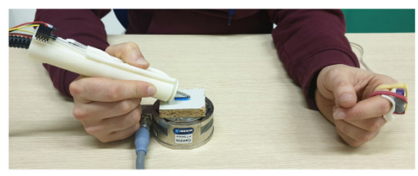

(c)

Fig. 3. (a) Rendered 3D model of the device; (b) mechanism for pulling up/down the fabric belt. (c) A user testing the proposed system.

We validated this method comparing the estimated contact force $F_{\text {est }}$ to the norm of the forces measured by the ATI in 200 contact actions involving both the z-axis and the xy-plane. The resulting RMSE is $0.967 \mathrm{~N}$, in a force range of $[0-18]$ N. Steps from pressure raw data toward force values are depicted in Fig. 2a. Once validated the sensing device in non-vibrating trials, we tested the proposed system switching on the drill. Adding vibrations introduces a significant modification in the force profile, as depicted in Fig. 2b. To compensate this negative effect, a software filter has been implemented. The filtered force value corresponds to the maximum value in a moving window of $33 \mathrm{~ms}$. In this way, the downward peaks are ignored guaranteeing a safer overestimation. This implies that it is not possible to compare the filtered force estimation with the measurements of the ATI in drilling tasks. The duration of the moving window was selected to obtain the best compromise between filter performance and response delay. All the steps of the force estimation are reported in Fig. 2c.

\section{Force Feedback}

Contextually with the force sensing system, we developed a haptic ring capable of generating cutaneous and vibrotactile force feedback. To have a lightweight device with a limited encumbrance, we employed a single servo-motor (HS-35HD Ultra Nano, HITEC Inc., USA) controlling a flexible belt for generating cutaneous stimuli and an eccentric-mass motor (EMM) to generate vibrotactile stimuli [7]. The device is controlled by the same DAQ board used for sensors data acquisition through an ad-hoc library. The servo motor generates the rotation of a master gear that moves a slave gear. Such mechanism results in opposite spinning directions of the gears, that translate the belt along the vertical axis. The workings are depicted in Figs. $3 \mathrm{a}$ and $3 \mathrm{~b}$. The maximum range of the belt motion in the vertical direction is $23 \mathrm{~mm}$ and it depends on the external diameter of the gears $(11 \mathrm{~mm})$, the length of the belt $(95 \mathrm{~mm})$, and the maximum rotation range of the servo motor $\left(120^{\circ}\right)$. We selected these values considering that also fingertips bigger than the average should fit. The maximum exploitable displacement range for force generation is $6 \mathrm{~mm}$, so that the device can apply a maximum force of $3 \mathrm{~N}$ considering a stiffness of $0.5 \mathrm{~N} / \mathrm{m}$ as elastic behavior of the finger pulp. Interested readers are referred to [6] and [4] for further details on the force feedback generation. A manual calibration is performed for each 
participant to adjust the initial position of the belt. The vibrational motor is placed horizontally alongside the device. It generates vibrations ( $1 \mathrm{~g}$ at $3.6 \mathrm{~V}$ ) to notify the force threshold over-reaching.

\section{Experimental Validation}

The experimental evaluation was carried out with a twofold aim: $i$ ) demonstrating the effectiveness of the haptic feedback in the aforementioned surgery and ii) identifying the best feedback approach. Ten users (6 males, age 23-56, all right handed) took part in the experiment. One was a surgeon with many years of experience, three were medical students with 5 years of experience, while the remaining six were medical students with lower/no experience in performing open surgical procedures.

The experiment aimed at simulating a cochlear implant surgery. Participants were asked to completely remove a blue colored rectangle $(0.6 \mathrm{~cm} \times 2.0 \mathrm{~cm})$ from a piece of plywood using the instrumented drill (rotating at $15.000 \mathrm{rpm}$ ). Users wore the haptic ring on the left hand (see Fig. 3c), where a clear perception of the haptic feedback is allowed by the absence of vibrations. Participants were told that the task was considered successfully accomplished when the drilling force was maintained in a specific range, i.e. $[0-7.5] \mathrm{N}$, without overreaching the limit time of $13 \mathrm{~s}$. The time limit has been introduced to prevent subjects from being excessively slow in order to completely remove the blue color using low forces. Three feedback conditions were evaluated: $i)$ no feedback $(N)$; ii) vibratory $(V)$ alert in case of exceeding the force threshold; iii) vibratory alert and cutaneous feedback proportional to the exerted force $(C)$. A proportional scale factor was used to map the measured maximum force into the admissible range of the ring. Each user performed a set of three trials per each feedback condition (pseudorandomly selected), resulting in a total of 9 trials. Time to complete the task and impulse (the integral of the force out of the boundaries over the time interval) were considered as metrics for evaluating the task performance. A familiarization period of 2 min was provided to acquaint participants with the system. In this phase, users tested the overreaching of the force limit with and without haptics. In the first case, the exerted force was displayed by the haptic ring, in the latter by a graphical indicator on a LCD screen.

\subsection{Results and Discussion}

Data collected in the experimental phase were analyzed by means of statistical tests. For each participant completion time and impulse were computed (see Figs. $4 \mathrm{~b}$ and $4 \mathrm{c}$ ). All the participants were able to completely remove the blue color in the time limit of $13 \mathrm{~s}$ both with and without the haptic feedback. The average completion time among all the trials is $10.85 \pm 2.54 \mathrm{~s}, 10.23 \pm 1.72 \mathrm{~s}$, $9.32 \pm 1.16 \mathrm{~s}$ for $N, V$, and $C$ feedback conditions, respectively. Statistical analysis revealed that there is no statistically significant difference in the completion time of the task using different feedback. As shown also in Fig. 4a-upper, the 


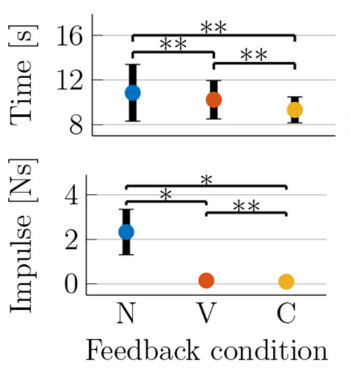

(a)

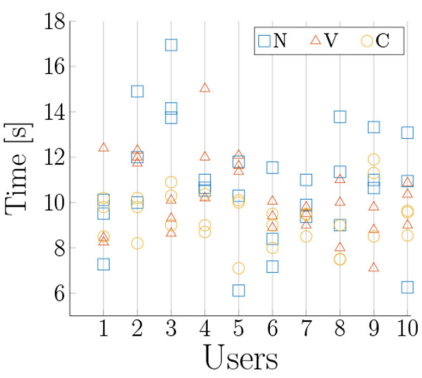

(b)

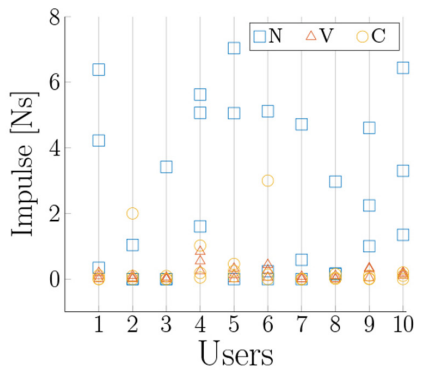

(c)

Fig. 4. In (a) mean and 95\% CI for all the feedback conditions are reported for time (upper panel) and impulse of the force over the threshold (lower panel). The $p$-values are reported on top of the error bars, ** and $*$ indicate $p>0.05$ and $p<0.0005$, respectively. In (b) and (c) users' results for each trial are shown.

task execution is not slowed down by the increasing number of stimuli to be focused on. Concerning the impulse of the force over the threshold, participant exceeded the limit with $2.33 \pm 1.25 \mathrm{Ns}, 0.15 \pm 0.16 \mathrm{Ns}, 0.10 \pm 0.13 \mathrm{Ns}$ testing the setup with $N, V$, and $C$ feedback, respectively. Moreover, a one-way repeated measures ANOVA was conducted to determine whether there were statistically significant differences in impulse over the different feedback. There were no outliers. Data were transformed using the squareroot transformation and passed the ShapiroWilk normality test $(p>0.05)$. The assumption of sphericity was violated, as assessed by Mauchly's test $\left(\chi^{2}(2)=7.87, p<0.05\right)$. Therefore, a Greenhouse-Geisser correction was applied. The results of the test (reported in Fig. 4a-lower) assessed that the feedback modality elicited statistically significant changes in over-applied forces $(p<0.0005)$. Post hoc analysis with Bonferroni adjustment revealed that the reduction of impulse was statistically significant. More in detail, the test revealed that there is statistically significant difference in performing the task with or without haptic feedback. In case of feedback, the impulse error had a almost complete reduction, which implies a more controlled penetration in the plywood. For what concerns the difference between the two haptic feedback, the difference is lower, but not statistically significant. Supported by the outcomes of the statistical analysis, we can affirm that haptic feedback can enhance the safety in surgical hand-held drilling tasks, maintaining the drilling force in a specific range. In addition, participants to the experimental campaign reported positive qualitative feedback on the haptic-assisted experience and on the positioning of the ring in the contralateral side. They argued that the cover did not interfere with the task and it would be useful to introduce the device in real surgical procedures, after appropriate refinements. 


\section{Conclusion and Future Work}

In this work, we presented a novel approach to measure the force exerted on bones during drilling tasks in open surgery. A pneumatic sensing cover for drills and a haptic ring to reproduce such forces were developed. We tested our sensing device in a comparison with a high-resolution/accuracy commercial force sensor, demonstrating the robustness of our approach. To properly reconstruct the force profile, we implemented both a hardware and a software filters. The advantage of our sensing system is that it can be easily adapted to any surgical drills, changing only few design parameters in the CAD model (e.g. introducing some shims to modify only the internal profile of the inner shell). The resolution and the range of our sensor are customizable: they can be modified changing the silicone pipes and using pressure sensors with different resolution.

We evaluated the effectiveness of our haptic-assisted hand-held drill with long experience and novice surgeons. Forces and vibrations were exploited to help the users in evaluating the real exerted forces. We compared the performance of the participants with and without haptic feedback, proving that haptic enhancement outperformed the haptic-free technique.

The presented results pave the way for numerous interesting research directions that will be the subject for future works. Different feedback policies and locations will be tested in a future experimental campaign. Additional metrics, such as tissue discrimination, will be considered to evaluate device and feedback. Learning curves in performing the task with and without feedback will be evaluated in a more careful future study, involving a larger sample. In further developments, the cover can be instrumented with additional sensors (e.g. an accelerometer) to measure the inclination of the drill and improve the calibration procedure. Finally, ergonomic studies will be taken into consideration.

\section{References}

1. Allotta, B., Giacalone, G., Rinaldi, L.: A hand-held drilling tool for orthopedic surgery. IEEE/ASME Trans. Mech. 2(4), 218-229 (1997)

2. Gaudeni, C., Meli, L., Prattichizzo, D.: A novel pneumatic force sensor for robotassisted surgery. In: Prattichizzo, D., Shinoda, H., Tan, H.Z., Ruffaldi, E., Frisoli, A. (eds.) EuroHaptics 2018. LNCS, vol. 10894, pp. 587-599. Springer, Cham (2018)

3. Hessinger, M., Hielscher, J., Pott, P.P., Werthschützky, R.: Handheld surgical drill with integrated thrust force recognition. In: Proceedings of IEEE International Conference on E-Health and Bioengineering, pp. 1-4 (2013)

4. Lisini Baldi, T., Scheggi, S., Meli, L., Mohammadi, M., Prattichizzo, D.: GESTO: a glove for enhanced sensing and touching based on inertial and magnetic sensors for hand tracking and cutaneous feedback. IEEE Trans. Human-Mach. Syst. 47(6), 1066-1076 (2017)

5. Louredo, M., Diaz, I., Gil, J.J.: DRIBON: a mechatronic bone drilling tool. Mechatronics 22(8), 1060-1066 (2012)

6. Park, K.H., Kim, B.H., Hirai, S.: Development of a soft-fingertip and its modeling based on force distribution. In: Proceedings of the IEEE International Conference on Robotics and Automation, vol. 3, pp. 3169-3174 (2003) 
7. Precision Microdrives: Model No. 304-002 4mm Vibration Motor - 8mm Type Datasheet. https://www.precisionmicrodrives.com/product/datasheet/304002-4mm-vibration-motor-8mm-type-datasheet.pdf

8. Sang, H., Monfaredi, R., Wilson, E., Fooladi, H., Preciado, D., Cleary, K.: A new surgical drill instrument with force sensing and force feedback forrobotically assisted otologic surgery. J. Med. Dev. 11(3) (2017)

9. Wagner, C.R., Stylopoulos, N., Howe, R.D.: The role of force feedback in surgery: analysis of blunt dissection. In: Proceedings of the IEEE Haptics Symposium, pp. 68-74 (2002)

Open Access This chapter is licensed under the terms of the Creative Commons Attribution 4.0 International License (http://creativecommons.org/licenses/by/4.0/), which permits use, sharing, adaptation, distribution and reproduction in any medium or format, as long as you give appropriate credit to the original author(s) and the source, provide a link to the Creative Commons license and indicate if changes were made.

The images or other third party material in this chapter are included in the chapter's Creative Commons license, unless indicated otherwise in a credit line to the material. If material is not included in the chapter's Creative Commons license and your intended use is not permitted by statutory regulation or exceeds the permitted use, you will need to obtain permission directly from the copyright holder.

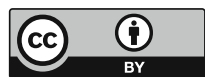

